\title{
Manager Interaction and the Formation of Strategic Consensus: The Moderation Effect of Manager Role
}

\author{
Zhang Ping \\ School of Business Administration, South China University of Technology, Guangzhou, China
}

\section{Email address:}

Bmzhp328@126.com

\section{To cite this article:}

Zhang Ping. Manager Interaction and the Formation of Strategic Consensus: The Moderation Effect of Manager Role. Science Innovation. Vol. 7, No. 4, 2019, pp. 115-119. doi: 10.11648/j.si.20190704.12

Received: August 20, 2019; Accepted: October 16, 2019; Published: October 23, 2019

\begin{abstract}
In the dynamic environment the role of middle managers is becoming increasingly important in strategic management. From the perspective of middle managers, Using the method of theoretical deduction, this thesis analyses the influence of interaction between top management team and middle managers on the formation of strategic consensus and the moderation effect of manager role on integrating the theories of top management team and middle managers. This study concludes that the more sufficient information exchange between top management team and middle managers, and the greater the cross-influence between top management team and middle managers, the higher the strategic consensus. And the participatory leadership of top management team and the active participation of middle managers play a positive moderating role in the relationship between managers' interaction and strategic consensus.
\end{abstract}

Keywords: Manager Interaction, Manager Role, Strategic Consensus

\section{管理者互动与企业战略共识的形成：管理者角色的调节作用}

\section{张平}

华南理工大学工商管理学院, 广州, 中国

\section{邮箱}

Bmzhp328@126.com

\begin{abstract}
摘要：动态环境下，企业中层管理者在战略管理中的作用日益重要。本研究从中层管理者视角出发，运用理论演绎的 方法，整合高层管理团队和中层管理者的理论，多层次、多角度地考察和分析中国企业高层管理团队与中层管理者的 互动对战略共识的形成的影响以及管理者角色在其中所发挥的作用。本研究认为高层管理团队与中层管理者的信息交 换越充分，高层管理团队与中层管理者的交叉影响越大，战略共识越高。而高层管理团队参与式领导、中层管理者的 主动参与在管理者互动与战略共识的关系中发挥了正向的调节作用。
\end{abstract}

关键词：管理者互动，管理者角色，战略共识

\section{1. 引言}

企业管理者，包括高层管理团队（TMT，Top Management Team) 和中层管理者（MMs, Middle Managers），在企业战略的制定和实施过程中发挥了重要
的影响[1]。高层管理团队制定企业战略方案并主导其实施, 中层管理者是指那些直接由高层管理团队指挥的又高于 一线管理人员的管理者, 是组织内沟通上下的“连接者”。 自Hambrick和Mason提出高层梯队理论以来, 关于高层管 理团队的研究取得了大量的成果[2]。而关于中层管理者的 
研究, 虽然一些学者探讨了中层管理者与组织学习与创新、 战略执行和战略制定过程等的关系, 但整体研究成果相对 来说还是显得很单薄[3]。

动态环境下, 中层管理者在战略实施过程中的作用越 来越突出, 需要加强对其的研究。随着中国企业外部环境 不确定性的日益加剧, 对于企业战略实施的有效性、主动 性和全员性都有了更高的要求。中层管理者在组织架构上 表现为是连接高层管理者与基层人员的桥梁, 在战略制定 和实施过程中承担了提供预选方案、收集信息、促进适应 和实施战略等多种角色 [4], 需要发挥其更大的主动性和决 策性作用，在企业战略管理中的作用日益重要和突出。麦 肯锡公司的研究表明中层管理人员已成为决定企业战略 成败的关键因素。

管理者战略共识直接决定了战略的成功与否。但在中 国企业内部战略共识“缩水”现象严重, 战略在向中下层员 工和外部利益相关者传递过程中共识程度逐步下降, 导致 精心制定的战略并不能得到有效执行 [5]。在企业达成战略 共识以及战略实施过程中, 作为制定企业战略的高层管理 团队发挥了 “领头雁”的作用, 而作为承上启下者的中层管 理者发挥了“脊梁”的作用, 一旦他们对于既定战略缺乏共 享式理解，会对企业战略执行造成严重障碍 [6]。

高层管理团队和中层管理者都是企业战略管理的行 为主体, 冊庸置疑, 两者的共同作用对提高战略实施的效 率和有效性及其重要, 但已有的研究者还只是沿着两条路 线在走, 分别探讨它们在战略管理过程中承担的不同角色 和发挥的不同作用 [7], 较少研究两者的共同作用与战略制 定与实施效果的关系。高层管理团队和中层管理者之间不 完全是领导和下属的关系, 它们之间的互动是一个交换信 息、相互影响、达成共识的过程, 在企业战略实施过程中 发挥了重要作用。因此, 我们需要开展管理者互动对战略 共识形成的影响以及管理者角色在其中所发挥角色的研 究, 以期透彻理解战略共识的内在机理。本论文运用理论 演绎的方法, 整合多种理论, 探讨高层管理团队与中层管 理者的互动与企业战略共识形成之间的关系, 以及管理者 角色在其中所发挥的调节作用, 将弥补已有研究的不足, 研究结果对企业战略管理实践具有指导作用。

\section{2. 理论基础}

\section{1. 战略共识}

战略共识是各级员工对本企业战略的理解程度、认同 程度和执行意愿, 是影响企业战略执行力和战略绩效的重 要因素 [8]。目前研究的重点主要集中在共识内容、共识主 体以及战略共识的后果上。对于战略共识的内容, 由于视 角的不同而造成内容的差异, 有的着眼于战略目标、手段 和对环境的感知, 更多的是理解为企业战略重点 [9、10]。

战略共识的主体从最开始的企业高层管理团队, 后逐 渐扩展到中下层管理者、全体员工, 甚至企业的外部利益 相关者 [10]。Dess和Priem认为战略共识是企业高管层或核 心人员关于企业目标、竞争途径以及环境感知等方面的一 致看法 [8]。Neilson将战略共识的范围从企业内部拓展到
了企业外部, 指出, 有效地执行战略还需要在组织外部建 立相应的共识, 以利于与关键的外部利益相关者合作 [9]。

战略共识的后果主要是指其对绩效及战略执行的影 响, Bourgeois对12家非多元化企业的研究表明, 高管团队 就企业目标和实现手段达成的共识与企业绩效正相关, 但 在没能就实现手段达成共识的情况下, 仅就企业目标达成 的战略共识与企业绩效负相关 [10]。Dess的研究却得出了 不同的结论, 认为就企业目标或实现手段达成的共识都与 企业绩效正相关，但就企业目标和实现手段同时达成的共 识与企业绩效的相关程度并没有显著提高[11]。有学者试 图在战略共识与企业绩效的关系研究中引入调节变量以 期廓清不同情景下战略共识影响企业绩效的机理, Priem 认为企业经营环境的动态性对战略共识与企业绩效之间 的关系起到正向调节作用, 即经营环境越是趋于动态, 战 略共识与企业绩效之间的正相关性就越显著 [12]。相对于 对企业绩效的影响而言, 战略共识应该对战略执行产生更 加直接和显著的影响。

\section{2. 高层管理团队的视角}

从高层管理团队视角探讨高层管理团队对企业战略 的影响, 是学术界研究的热点, 取得了丰硕的研究成果, 已有的研究集中在四个方面, 分别是:

（1）高层管理团队特征与战略选择、企业绩效的关 系。主要是探讨高层管理团队的传记性特征及其异质性、 断裂带等对组织产出的影响, 认为高层管理团队成员的年 龄、教育水平、任期、职业经验、性别等与他们对环境的 洞察力密切相关, 并决定了所采取的战略行动, 进而影响 了组织的绩效。研究中选取的 TMT变量主要是其年龄、 教育、职业经验、任期等平均值或异质性, 高层管理团队 的异质性是指团队成员间传记性特征的差异化程度。该方 面的研究, 成果很多, 但实证研究并没有给出一个一致性 的结果, 如有学者发现, 中国上市公司的高层管理团队的 任期异质性、职业经验异质性与企业绩效负相关 [13]。企 业高层管理团队内存在各种断裂带, 有些甚至是天然的, 如年龄、国别、性别等。团队成员在一个或多个属性上的 差异像是一条虚拟的线, 会将团队分成不同的子团队, 这 条虚拟的线就被称为团队断裂带[14]。有学者将团队断裂 带模型引入到高层管理团队的研究中, 探讨高管团队断裂 带与企业战略和绩效的关系。Ndofor等的研究发现, 当团 队异质性强化成为断裂带时, 企业资源和竞争行动间的正 向关系不显著了 [15]。

(2) 高层管理团队运作过程与战略选择、组织绩效。 打开高层管理团队 “黑箱”, 探讨团队内部的权力结构、冲 突、一致性、行为整合等运作过程对企业战略、绩效的影 响。Greve和Mitsuhashi研究发现领导权集中有利于高管团 队在决策过程中减少花费在信息交流和争论上的时间[13], 而Smith等的研究表明高管团队领导权内部分布不均衡会 导致战略决策备选方案的数量和质量无法得到保证, 还会 影响战略决策的实施速度 [16]。团队的行为整合是指高层 管理团队成员在思想和行动上的集体互动, 这种互动包括 信息交流的数量和质量、合作行为以及共同决策，对于提 
高团队效能具有积极的作用。刘金的调查研究发现, TMT 行为整合对组织双元性有显著地积极影响 [17]。

(3) 高层管理团队认知/价值观与战略选择、企业绩 效。随着测量认知、价值观的工具不断被开发出来, 国内 外的学者开始重视直接研究高层管理团队成员认知/价值 观、认知异质性等对企业战略的影响, 而不是采用高层管 理团队传记性特征这个代理变量。Miller等通过三个独立 的研究, 指出高管认知的多样性阻碍了对机会、威胁的分 析全面性, 以及降低了战略计划的广泛性, 而企业战略的 全面性和广泛性与绩效正相关[13]。白云涛、郭菊娥和席 西民采用针对MBA学员的实验方法, 发现, 高层管理团 队的风险偏好异质性与成员的过程和结果满意度有负相 关关系，与决策时间有正相关关系，与决策一致性具有正 “U”型的相关关系[18]。

（4）跨文化的研究。高层管理团队的认知、价值观 和决策会受到其嵌入的社会文化环境影响, 一些学者研究、 比较不同国家中高层管理团队与企业战略决策关系的异 同点。Glunk、Heijltjes和Olie对瑞典、荷兰的跨国公司TMT 组成中国籍多样性的研究发现, 虽然跨国公司的国际化程 度不断增加, TMT中拥有国外成员的公司的比例以及国外 TMT成员的比例远低于企业的国际化程度 [19]。Crossland 和Hambrick的研究表明, 美国企业的高管团队对公司绩效 的影响要显著高于德国、日本公司[13]。

\section{3. 中层管理者的视角}

中层管理者在企业战略管理过程中作用的研究, 取得 了一些成果, 这些研究加深了我们对战略实施的理解, 它 们被称之为“中层管理者视角 (Middle Management Perspective）”[20]。中层管理者视角的研究主要围绕中层 管理者的战略角色和前因、中层管理者的战略参与和组织 认知等展开。

（1）中层管理者的战略角色和前因。中层管理者角 色可以被分为四类[21]: 实施者, 这是传统的角色, 主要 的作用是其行为与现有的战略保持一致, 并围绕这一战略 整合下属的行为; 综合者, 中层管理者解读信息并向上传 递; 向上发动者, 影响高层管理团队, 中层管理者的发散 性思维可能重塑他们对现有战略的理解; 推动者, 中层管 理者鼓励下属积极思考和勇于创新。有些实证研究证实了 中层管理者的某一类角色, Dutton等、Ling等的研究表明 中层管理者是一个“主题推介员 (Issue-selling Role) ”, 这 是综合者和向上发动者角色的合一 [22、23]。通过主动向 高层管理推介他们感兴趣的主题, 中层管理者改变了企业 的战略计划。关于中层管理者角色的前因变量, Ling等的 研究表明中层管理者的向上主题推介和包装策略受不同 国家民族文化的影响 [23]。Marginson指出管理控制系统会 改变中层管理者的战略角色 [24]。卡尼则发现组织结构, 特别是管理层级的多少影响中层管理者对战略角色的看 法, 并认为扁平的组织结构能够强化沟通, 促使中层管理 者积极参与战略 [6]。

（2）中层管理者的战略参与和组织认知。该部分研 究主要是分析中层管理者认知如何影响他们对战略的理 解以及参与战略的过程如何又影响了他们的认知。最初的
文献聚焦于战略共识, 而最新的研究集中在战略过程中如 何发展形成共享的战略思维。Floyd和Wooldridge认为除非 中层管理者理解并支持高层管理团队制定的战略目标, 否 则他们不会推进战略实施, 即使高层管理团队推动也难以 实现有效的战略变革 $[4]$ 。King和Zeithaml研究了中层管理 者的战略思维与企业竞争力的关系, 认为高层管理团队与 中层管理者共享思维与企业竞争力的难以模仿性正相关 [25]。

已有的关于管理者与战略共识形成的研究, 取得了大 量的成果, 加深了对战略共识的形成、中层管理者在其中 作用的认识, 但仍存在针对企业战略共识的研究不足, 无 论是研究成果的质量上还是数量上; 在关于战略共识的研 究中, 割裂了高层管理团队、中层管理者之间的关系, 而 形成共识是需要双方参与的; 也缺乏有深度的基于中国情 境的中层管理视角的企业战略共识形成的研究成果。

\section{3. 高层管理团队与中层管理者互动对战略共识 的影响}

企业战略是一个企业为适应环境变化而不断调整组 织行为的动态过程, 其成功的关键因素是参与战略制定与 实施的人员确信他们能持续的预见环境变化并将这些信 息有效地用于战略决策。中层管理者在这个过程中扮演了 重要的角色, 因为他们能够及时发现外部环境中的机会并 且由此而启动和拥护新的战略举措。但是, 中层管理者也 可能成为战略实施的消极因素, 特别是当他们接触一个新 战略, 他们不理解或是新战略措施损害其自身利益。因此, 为有效推动战略实施, 高层管理团队和中层管理者双方需 要很好的整合各自的信息, 形成战略共识并实现双赢。

由于高层管理团队和中层管理者都有繁重的管理工 作，且他们之间不是一般的“领导-下属”关系，他们之间不 是频繁、紧密的互动, 而是短暂的接触和无联系之间交替 进行, 因此, 他们之间的互动是需要抓住宝贵的“机会窗 口”并承担额外的加深理解、促进合作和调整行为的功能。 我们可以从信息处理理论 (Theories of Information Processing) 和战略实施的人际交往观 (Interpersonal View of Strategy Implementation）两个视角来分析高层管理团队 与中层管理者互动对战略共识的影响。

根据信息处理理论, 学者认为战略制定和实施的质 量来自于高层管理者收集的信息种类和数量以及在战略 决策过程中对它们的分析、综合和运用 [13]。已有的关于 高层管理团队研究表明, 高异质性的高层管理团队由于 有多样化的信息来源和更多的公开讨论, 其战略决策质 量更高。基于类似的逻辑, 由于中层管理者与高层管理 团队所处的位置不同, 导致信息来源存在差别, 而且两 者对同一信息还有不同的解释框架, 高层管理团队和中 层管理者的互动是双向的, 丰富了信息来源途径和提高 了解释质量, 更有利于形成战略共识。战略实施的人际 交往观认为所有参与战略实施人员间的交流和互动是成 功的关键 [26]。Jarzabkowsi和Spee认为战略是由组织行动 者间一系列的行动和互动组成的 [20]。在这些互动中, 高 层管理团队能够将企业战略的深层次理解、战略实施的 
要求顺利地传递给中层管理者, 并激励他们去更好的实 施战略。另一方面, 中层管理者可以通过互动, 将资源 诉求、部门目标及时上传, 并影响具体战略实施的计划 安排, 从而达成战略共识。因此, 高层管理团队与中层 管理者之间的互动主要是信息交换和交叉影响, 且高层 管理团队与中层管理者的信息交换越充分, 高层管理团 队与中层管理者的交叉影响越大, 战略共识越高。

\section{4. 管理者互动与战略共识：管理者角色的调节 作用}

高层管理团队与中层管理者的互动与战略共识的关系 同时还受到双方各自所处的角色的影响。角色理论（Role Theory）认为社会位置决定了角色行为, 嵌入在组织中的管 理者其行为受职位、个人期望和他人行为共同影响[26]。高 层管理团队和中层管理者各自的战略角色是基本清楚和明 确的, 高层管理团队的主要职责是战略制定, 而中层管理者 是战略实施, 尽管他们都参与了战略管理的整个过程。中层 管理者除了是公司战略实施主要人员外, 还是本单位的利益 代表, 这种角色的多重性使得高层管理团队的期望和中层管 理者的自我认知会产生一定的冲突, 影响战略共识的形成。 但高层管理团队与中层管理者的角色行为是一个动态演化 的过程, 当他们在互动中发现有角色和行为冲突时, 他们会 不断调整和更新自己的行为以实现新的协调, 也就是说, 一 方的角色行为能够塑造随后双方互动中的行为。

我们考察管理者角色在战略形成中的影响, 同时从高层 管理团队和中层管理者两个角度来进行分析。首先是高层管 理团队的参与式领导（Participative Leadership），这是指在 战略制定与实施过程中高层管理团队愿意积极寻求和利用 中层管理者的信息和建议[9]。参与式领导是一种基于对角色 相互理解的高层管理团队行为模式, 越高就意味着高层管理 团队越重视中层管理者信息和建议的价值, 经常与中层管理 者互动以获取这些信息, 并将其作为战略决策的基础。这提 高了管理者之间的战略共识并保证了战略的有效性和效率。 高层管理团队从其他层级获得的信息越多, 战略选择就越又 快又好。其次是中层管理者的主动参与 (Active Engagement), 这是指中层管理者有兴趣思考且积极推动对企业战略有利 的行为 [3]。当中层管理者主动参与到战略制定与实施过程中 来时, 他们更愿意积极地与高层管理团队互动, 主动提供更 多的信息, 主动分析和理解高层管理团队提出的建议并给出 具有建设性的替代方案。通过中层管理者的积极参与, 提高 了高层管理团队与中层管理者互动的效果, 使得他们对环境 的认识、方案的理解、实施的困难等都会有更深、更广的理 解, 有利于形成战略共识。因此, 可以说高层管理团队参与 式领导在管理者互动与战略共识的关系中发挥了正向的调 节作用; 中层管理者的主动参与在管理者互动与战略共识的 关系中发挥了正向的调节作用。

\section{5. 结论}

高层管理团队和中层管理者都是企业战略制定和实 施的重要成员, 他们的互动影响了战略共识的形成。本研
究认为高层管理团队与中层管理者之间的互动主要是信 息交换和交叉影响, 且高层管理团队与中层管理者的信息 交换越充分, 高层管理才队与中层管理者的交叉影响越大, 战略共识越高。而高层管理团队参与式领导在管理者互动 与战略共识的关系中发挥了正向的调节作用; 中层管理者 的主动参与在管理者互动与战略共识的关系中发挥了正 向的调节作用。

本研究整合高层管理团队和中层管理者视角, 从理论 上厘清了管理者互动、管理者角色和企业战略共识形成之 间的关系, 特别是深入探讨了中层管理者在战略共识形成 中所发挥的作用, 丰富了企业战略管理的研究内容。本研 究只是运用理论演绎得出结果, 后续可以开展利用二手数 据、问卷调查、案例研究等实证研究, 以取得有创新性的 成果。

\section{致谢}

本文受教育部哲学社会科学研究重大课题攻关项目 “中国制造业转型升级战略研究”(15JZD020)，教育部人 文社会科学研究一般项目“多重制度逻辑下中国企业海外 子公司合法性的构建机理研究”（19YJA630113），广东 省自然科学基金项目“管理者互动、战略共识与战略实施 效果的研究: 中层管理者网络位置的调节作用” （2017A030313404）, 华南理工大学中央高校基本科研业 务费专项资金“制度压力下我国企业政治关联、高层管理 团队认知与产业链整合战略选择的研究”（2015ZZ060）资 助。

\section{参考文献}

[1] Floyd S. W., Wooldridge B. Middle management involvement in strategy and its association with strategic type: A research note [J]. Strategic Management Journal, 1992, 13: 153-167

[2] Hambrick, D. C., Mason P. A., Upper Echelons Theory: An Update [J], Academy of Management Review, 2007, 32 (2): 334-343.

[3] Floyd S. W., Wooldridge B. Middle management involvement in strategy and its association with strategic type: A research note [J]. Strategic Management Journal, 1992, 13: 153-167.

[4] 薛云奎, 齐大庆, 韦华宁, 中国企业战略执行现状及执行 力因素分析 $[J]$, 管理世界，2005，9:88-98。

[5] Noble C.H. The Eclectic Roots of Strategy Implementation Research [J]. Journal of Business Research, 1999, 45: 119 134.

[6] 黄再胜, 战略共识理论研究述评 [J], 外国经济与管理, 2010,3: 11-17、39.

[7] Kellermanns F.W., Walter J., Lechner C., Floyd S.W., The Lack of Consensus aboutStrategic Consensus: Advancing Theory and Research [J]. Journal of Management, 2005, 31 (5): 719-737. 
[8] Dess G. G., Priem, R. L., Consensus-performance Research: Theoretical and Empirical Extensions [J]. Journal of Management Studies, 1995, 32 (4): 401-417.

[9] Nielsen R. P., Strategic Planning and Consensus Building for External Relations -- Five Cases [J], Long Range Planning, 1983, 16: 74-81.

[10] Bourgeois, L J. Performance and consensus [J]. Strategic Management Journal, 1980, 1 (3): 227-248.

[11] Dess, G G. Consensus on strategy formulation and organizational performance: Competitors in a fragmented industry [J]. Strategic Management Journal, 1987, 8 (3): 269-277.

[12] Priem R.L., Top Management Team Group Factors, Consensus, and Firm Performance [J]. Strategic Management Journal, 1990, 11: 469-478.

[13] 蓝海林, 我国上市公司高层管理团队异质性与企业绩效的 关系研究 [M], 经济科学出版社, 2005: 198-203.

[14] Lau D, Murnighan JK. Demographic diversity and faultlines: the compositional dynamics of organizational groups [J]. Academy of Management Review, 1998, 23 (2): 325-340.

[15] Ndofor H. A., Sirmon D., He X. M. Utilizing the Firm's Resources: How TMT Heterogeneity and Resulting Faultines Affect TMT Tasks [J]. Strategic Management Journal, 2015, 36: $1656-1674$.

[16] Smith K. G., Smith K. A., Olian J. D., Sims H. P., O’Bannon D. P., Scully J. A., Top management team demography and process: the role of social integration and communication [J], Administrative Science Quarterly, 1994, 39, 412-438.

[17] 刘金金, 蒋春燕, 高层管理团队行为整合对组织双元性的影 响: 战略决策周密性的中介作用及长期薪酬的调节作用 [J], 商业经济与管理，2015，7:25-33。
[18] 白云涛, 郭菊娥, 席西民, 高层管理团队风险偏好异质性 对战略投资决策影响效应的实验研究 $[\mathrm{J}]$, 南开管理评论, 2007，2: 25-30。

[19] Glunk U., Heijltjes M. G., Olie R., Design characteristics and functioning of top management teams in Europe [J], European Management Journal, 2001, 19 (3): 291-300.

[20] Wooldridge B., Schmid T., Floyd S. The Middle Management Perspective on Strategy Process: Contributions, Synthesis, and Future Research [J], Journal of Management, 2008, 12: 1190-1221.

[21] Floyd S. W., Wooldridge B., The Strategic Middle Manager: How to Create and Sustain Competitive Advantage [M]. San Francisco: Jossey-Bass, 1996: 48-51.

[22] Dutton J. E., Ashford S. J., O’Neill D. R., Lawrence K. A., Moves that Matter: Issue Selling and Organizational Change [J]. Academy of Management Journal, 2001, 44 (4): 716-736.

[23] Ling Y., Floyd S. W., Baldridge D. C., Reading the Winds in Multinational Corporations: The Impact of Culture on Issue Selling Behavior $[\mathrm{J}]$. Journal of International Business Studies, 2005, 36: 637-654.

[24] Marginson W., Management Control Systems and their Effects on Strategy Formation at Middle-management Levels: Evidence from a UK Organization [J]. Strategic Management Journal, 2002, 23: 1019-1031.

[25] King A. W., Zeithaml C.P., Competencies and the Causal Ambiguity Paradox [J]. Strategic Management Journal, 2001, 22: 75-99.

[26] Homburge, C, Krohmer, H, and Workman, J P. Strategic consensus and performance, the role of strategy type and market-related dynamism [J]. Strategic Management Journal, 1999, 20 (4): 339-357. 